\title{
A new image denoising model utilizing the conformable fractional calculus for multiplicative noise
}

\author{
Rabha W. Ibrahim ${ }^{1}$
}

Received: 11 September 2019 / Accepted: 17 November 2019 / Published online: 6 December 2019

(c) Springer Nature Switzerland AG 2019

\begin{abstract}
Reducing noise from images is an essential structure of the image processing study. Noises can arise with images through achievement on diffusion. The existence of noise can delay the right operation of these images for many applications such as satellite and medical images. Reducing denois in images multiplicatively (DIM) has been developed and modified by many researchers during the past few years. DIM can destroy almost all data of the original image, especially the texture of images. Our aim is to present a new technique to solve this problem. The technique is based on a new fractional calculus called the conformable fractional calculus (CFC). This type of calculus has advantages because of its formula involves a controller, which can be applied to complex problems such as DIM. The proposed structures of CFC windows are given by four masks suggested for $x$ and $y$ directions. On four directional angles, a convolution operational product of the input image pixels with a CFC mask window has been completed. The visual observation and peak signal-to-noise ratio with Root Mean Square Error are employed for measurements. The experiments showed that the skillful filtering outcomes are indicated high score than some well known filers such as Gaussian filter, Sobel edge filter, Canny edge filter and gray-level co-occurrence matrix. Compering is illustrated as well with newly researches.
\end{abstract}

Keywords Fractional calculus · Fractional operator · Fractional mask - Image denoising - Multiplicative noise Conformable calculus · Gamma function

\section{Introduction}

Denoising is a substantial dealing out instruction for numerous requests in all images studies such as segmentation, texture analysis, and feature extraction. Image processing involves a number of phases, where denoising of images is the first responsibilities to be assumed and the recent challenge in this direction of studies is denois in images multiplicatively (DIM). DIM simulations are dominant to the study of logical imaging structures [1,2], by means of SAR ( synthetic aperture radar), laser imaging and ultrasound imaging. Due to the logical environment of these image achievement procedures, the normal additive noise structure is insufficient for meting out such images. In its place, DIM simulations deliver a high score explanation of logical imaging structures. There are different techniques deal with removing DIM based on the diffusion equation formal, partial differential equations, variation methods, gradient operational method and fractional differential equations [2-5].

The fractional calculus (FC) is briefly suggested in all sciences, including social science, law and economy with applications for the last 5 decades. In computer science, it has been deliberated, especially in the field of image processing [6-10]. It has been employed in image processing for medical MR image enhancement (see for recent work [11]). Image noise mentions to any undesired signal that perverts images. Noise acts in digital images primarily by image achievement, during which an optical image is transformed into continuous electrical signals.

Rabha W. Ibrahim, rabhaibrahim@yahoo.com | ${ }^{1}$ Cloud Computing Center, University Malaya, Kuala Lumpur, Malaysia. 
With former effort, fractional Conway polynomials (using differential and integral fractional operators to generalize these polynomials) with normalized fractional power parameters have been suggested for image denoising [6]. The advantage of utilizing these polynomials is paid to its capability to professionally eliminate (remove) the image noise. In [7], an upgraded denoised procedure founded by fractional Alexander polynomials is offered for eliminating diverse kinds of image noise. Investigates consequences presented that these polynomials effectively removed the noise and enhanced the image information as well. In addition, image noise removal procedure utilizing both fractional Tsallis entropy with the fractional Riesz has been showed in [9]. The developments attained in eliminating image noise were well-matched with typical image denoing filters.

Multiplicative noise representations are essential to the study of logical imaging systems, such as synthetic crack sonar, radar and ultrasound and laser imaging. These representations familiarize two further layers of problems with respect to the regular Gaussian additive noise setting: firstly, the noise is multiplied by the creative image; secondly, the noise is not Gaussian, where Gamma actuality utilized compactness. In this investigation, we suggest a new method created by a special type of $\mathrm{FC}$, called conformable fractional calculus (CFC) involving Gamma function. The advantages of CFC are that its formulation includes a controller, which can be employed in a complex system such as images. The anticipated constructions of CFC windows are assumed by four masks suggested for $x$ and $y$ directions. Different filters are employed for measurements. The experiments showed that the skillful filtering outcomes are indicated a high score than some well-known filers.

The rest of this study is organized as follows: Section 2 is related works in multiplicative noise; Sect. 3 , the proposed model is explained. In Sect. 4, the experimental results are described. Finally, the comparison with other methods and conclusion are presented in Sects. 5 and 6 respectively.

\section{Related works}

Yousef and Reza [12] introduced an image denoising method based on the wavelet transform domain for multiplicative noise. This method did not involve prior exhibiting of either the image or the noise information. Later Rudin et al. [13] reserved optimization category of numerical procedure for restoring blurry, noisy images. The authors presented both theoretical and experimental explanation for the technique. Dias et al. [14] designed a system by employing the variable splitting and constrained optimization, while the Douglas-Rachford splitting methods are indicated by Steid Teuber [15]. Li and Xie applied the concept of fractional calculus of small probability strategy [16]. Other fractional approaches utilized by considering different types of diffusion equations, smooth diffusion equation, differential operators, integral operators of fractional order, fractional entropy and Wavelet energy entropy (see for recent efforts [17-20]). All these methods described fractional masks by using different values of gamma function and its compositions. Other fractional techniques formulated by using polynomials and series such as fractional Fourier transform (see [21-23]). In this place, we note that this is the first application of conformable fractional calculus in image processing. The important of this calculus is that one of its term is based on controller function (gradient); therefore, we include the gradient implicitly in the processing and analysis of the image. Moreover, the fractional power comes from two sides. The first one is by multiplying the image by a fractional function and the second by multiplying the gradient of the image by another fractional function. For extra works, we advice the reader to check [24-27].

Images detection and description from fixed-motivated and self-motivated acts is a dynamic stud and investigation of research and one of the most considered issues in computer vision works. The notion of image detection and description discusses to the progression of identifying points in an image (interest points) that can apply to describe the image's contents such as corners, edges, ridges and blobs (for review work see [28]). The skill of treating digital features kept within the computer developed an important technology in numerous arenas and employed as a fundamental measure in an outsized number of manufacturing vision applications (see Hassaballah et al. [29]). Moreover, Hassaballah et al. [30] discussed the size of digital feature sources continue to develop, with the progress of the Internet, reduction in data-storage costs, and developments in expertise for feature-capturing strategies.

The shape contents is one of the important factor in feature deblurring. The image formula is generally imposed by $B=l * k+\varepsilon$, where $B$ is the blurred input image, $l$ is the latent sharp image, $k$ is the blur kernel, $*$ is the convolution operator, and $\varepsilon$ is the noise term [31]. Since our algorithm based on convolution property, then we can say that the proposed method can determine and cover this factor. Ancuti et al. [32] introduced another study in image processing describing the hand-designed features, such as the dark channel, color disparity and maximum contrast, with complex synthesis structures, limit the presentation of remaining image dehazing systems.

Finaly, different methods of image processing developed and presented; for example Khan et al. [33] presented a method by using particle swarm optimization, Nazir et al. [34] created a histogram of oriented gradients based on transformed features and Munir et al. [35] merged different methods to obtain good results. 


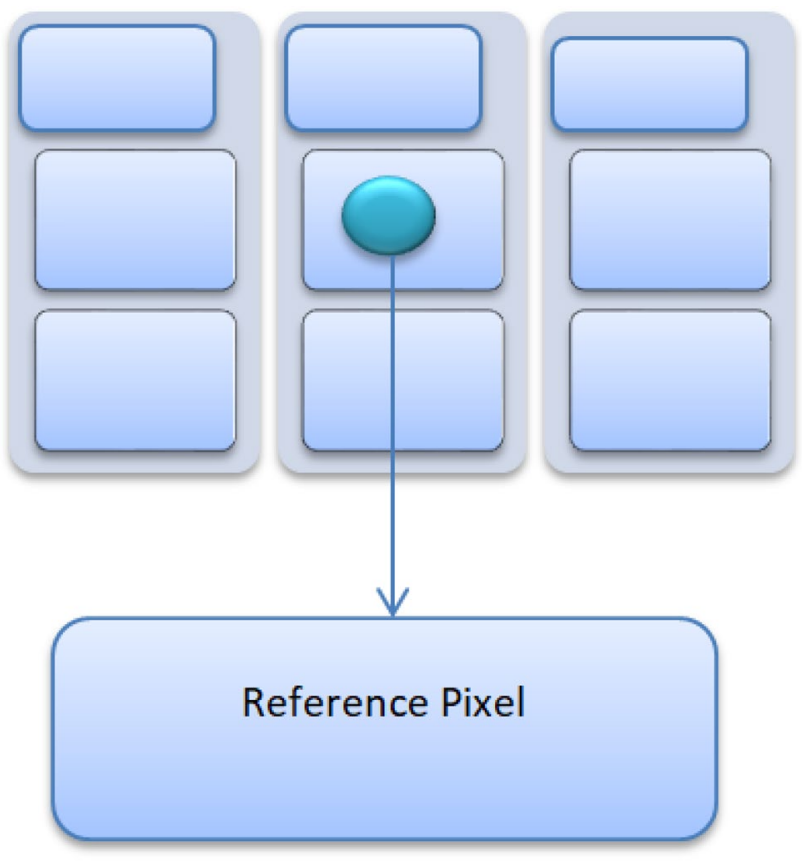

Fig. 1 The first type method in image denoising

\section{CFC model}

The noise smooth in digital images may differ from organism practically invisible to organism obvious. Image denoising procedures is aim to yield a new image that has less noise, i.e., closer to the novel noise-free image. Image denoising methods can gather into two central methods: pixel-based image filtering and patch-based filtering. The first approach is a proximity operation utilized operating one pixel at a based on its 3-D neighboring pixels situated inside a kernel. While, the second type is using "blocks," which are then operated distinctly in order to deliver an estimate of the exact pixel prices based on similar bits situated within a examine window. This method operates the redundancy and the likeness among the numerous shares of the effort image (see Figs. 1 and 2 respectively). Our method can recognize the second type. CFC is an extension of differentiation and integration of the functions defined on fractal sets. The notion of CFC has been a subject of attentiveness, not only among mathematicians, but also among engineers and scientists. There are many forms of CFC derivatives and CFC integrals [36-38]. The proposed model is based on Anderson-Ulness definition [36].

Let $\alpha \in[0,1]$, then a differential operator $\mathcal{D}^{\alpha}$ is conformable if and only if $\mathcal{D}^{0}$ is the identity operator and $\mathcal{D}^{1}$ is the classical differential operator. Specifically, $\mathcal{D}^{\alpha}$ is conformable if and only if for differentable function $f=f(t)$,

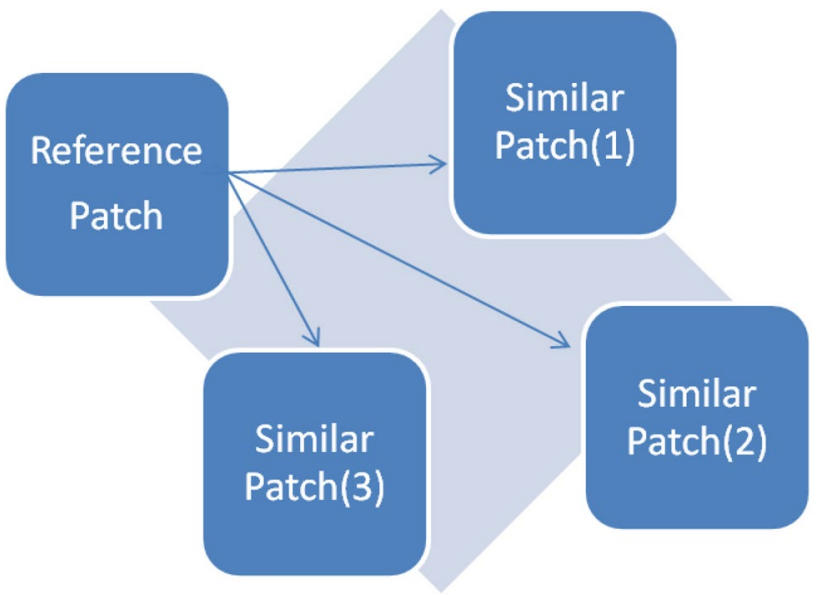

Fig. 2 The second type method in image denoising

$\mathcal{D}^{0} g(t)=g(t)$ and $\mathcal{D}^{1} g(t)=\frac{d}{d t} g(t)=g^{\prime}(t)$.

In [36] the authors noted that in control theory, a proportional-derivative controller for controlling output $\mu$ at time $t$ with two tuning parameters has the algorithm

$\rho(t)=\sigma_{p} \Upsilon(t)+\sigma_{d} \frac{d}{d t} \Upsilon(t)$,

where $\sigma_{p}$ is the proportional gain, $\sigma_{d}$ is the derivative gain, and $Y$ is the error between the state changing and the process changing. From the controller $\rho$, Anderson and Ulness introduced the following CFC.

Definition 1 Assume that $\alpha \in[0,1]$ and two continuous functions $\sigma_{0}, \sigma_{1}:[0,1] \times \mathbb{R} \rightarrow[0, \infty)$ achieving the approximations

$\lim _{\alpha \rightarrow 0} \sigma_{1}(\alpha, x)=1, \quad \lim _{\alpha \rightarrow 1} \sigma_{1}(\alpha, x)=0$,

$\sigma_{1}(\alpha, x) \neq 0, \forall x \in \mathbb{R}, \alpha \in(0,1)$,

and

$\lim _{\alpha \rightarrow 0} \sigma_{0}(\alpha, x)=0, \quad \lim _{\alpha \rightarrow 1} \sigma_{0}(\alpha, x)=1$,

$\sigma_{0}(\alpha, x) \neq 0, \forall x \in \mathbb{R} \alpha \in(0,1)$.

Then for a differential function $\Upsilon$ the following CFC is given

$D^{\alpha} \Upsilon(x)=\sigma_{1}(\alpha, x) \Upsilon(x)+\sigma_{0}(\alpha, x) \Upsilon^{\prime}(x)$,

where $\Upsilon$ is the true image, $x$ is the pixel, $\sigma_{1}, \sigma_{0}$ are the multiplicative and additive noise components, respectively, and $D^{\alpha} \Upsilon$ is is the observed image.

In imaging, we refer to $\Upsilon^{\prime}(x)$ as the discrete Laplaciana operator (or its average formal operator, which converges to 
the solution $\Upsilon$ ). Thus, as a conclusion, we shall use symbolized CFC formal by

$D^{\alpha} \Upsilon=\sigma_{1}^{\alpha} \Upsilon+\sigma_{0}^{\alpha} \Delta \Upsilon \approx\left(\sigma_{1}^{\alpha}+\sigma_{0}^{\alpha}\right) \Upsilon$.

\subsection{Construction of CFC filter}

To construct, the CFC filter, we assume that

$\sigma_{1}^{\alpha}(l, J)=\frac{1-\alpha}{\Gamma(l+J-\alpha)}, \quad \sigma_{0}^{\alpha}(l, J)=\frac{\alpha}{\Gamma(l+J-\alpha)}, \quad l, J=1,2,3, \ldots$

It is clear that $\sigma_{1}, \sigma_{0}$ satisfy Definition 1 . Let $\sigma^{\alpha}(l, J):=\sigma_{1}(l, J)+\sigma_{0}(l, J)$. Since the image is a function of two variables; therefore, we extend $D^{\alpha} \Upsilon$ into two variables to get

$D^{\alpha} \Upsilon(x, y)=\sum_{l} \sum_{j} \sigma^{\alpha}(l, J) \Upsilon(x+l, y+J)$,

with the coefficients

$$
\begin{aligned}
\sigma^{\alpha}(l, J) & =\frac{1-\alpha}{\Gamma(l+J-\alpha)}+\frac{\alpha}{\Gamma(l+J-\alpha)} \\
& =\frac{1}{\Gamma(l+J-\alpha)} .
\end{aligned}
$$

These coefficients are used to enhance the images. The CFC for image denoising is $\Upsilon_{d}$, which is computed from input noising image $\Upsilon_{n}$ :

$\Upsilon_{d}(x, y)=\sigma^{\alpha} * \Upsilon_{n}(x, y)$,

where $\sigma$ is the window's mask, and ${ }^{*}$ is the convolution product. The CFC-filter window's mask is given by the coefficients $\sigma^{\alpha}(l, J)$. In this note, four masks are systematized as follows:

$\omega_{0^{\circ}}=\left[\begin{array}{ccc}0 & 0 & 0 \\ \sigma^{\alpha}(2,1) & \sigma^{\alpha}(2,2) & \sigma^{\alpha}(2,3) \\ 0 & 0 & 0\end{array}\right]$,
$\omega_{45^{\circ}}=\left[\begin{array}{ccc}0 & 0 & \sigma^{\alpha}(1,3) \\ 0 & \sigma^{\alpha}(2,2) & 0 \\ \sigma^{\alpha}(3,1) & 0 & 0\end{array}\right]$

$\omega_{90^{\circ}}=\left[\begin{array}{lll}0 & \sigma^{\alpha}(1,2) & 0 \\ 0 & \sigma^{\alpha}(2,2) & 0 \\ 0 & \sigma^{\alpha}(3,2) & 0\end{array}\right]$,

$\omega_{135^{\circ}}=\left[\begin{array}{ccc}\sigma^{\alpha}(1,1) & 0 & 0 \\ 0 & \sigma^{\alpha}(2,2) & 0 \\ 0 & 0 & \sigma^{\alpha}(3,3)\end{array}\right]$.

The pixel values of CFC window's mask are computed by shifting the window's mask over the input image. One of the advantages of the proposed filter is that CFC, has a mathematical ability to consider the additive noise as a multiplicative noise (see Eq. 3.7). This behavior allows CFC to follow a gamma distribution [24]. The proposed CFC window's mask image denoising can be summarized as follows (see Fig. 3):

- Begin CFC window's mask utilizing $3 \times 3$ pixel measure;

- Give the adequate values of $\alpha$ with the range of $0<\alpha \leq 1$. We found a good result when $\alpha \in(0.5,0.75)$ (see Fig. 4);

- Employ CFC in four directions over the input image;

- Utilize any well know filter, such as GSF, SEF, CEF, for comparison;

- Calculate the PSNR, and SSIM for all filters including CFC-filter.

For color images, the same algorithm is suggested and for gray-scale images is applied separately for each components.

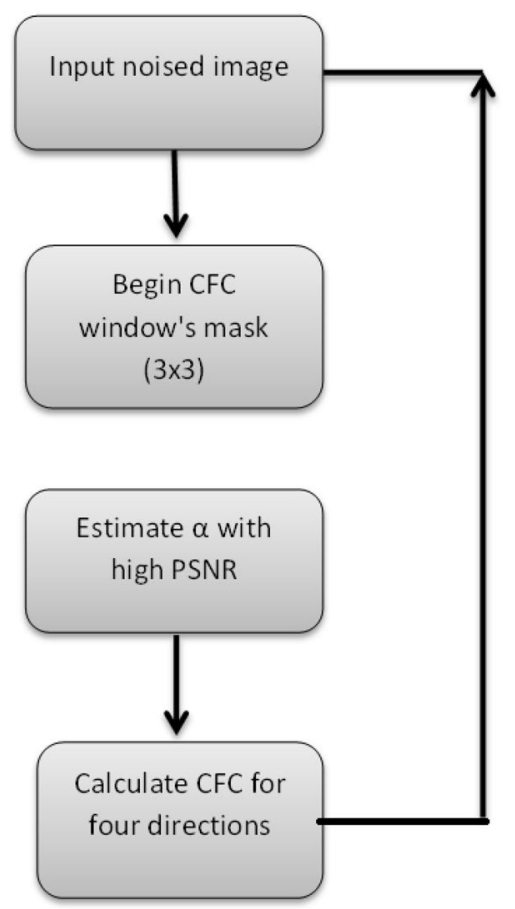

Fig. 3 The algorithm of using CFC 

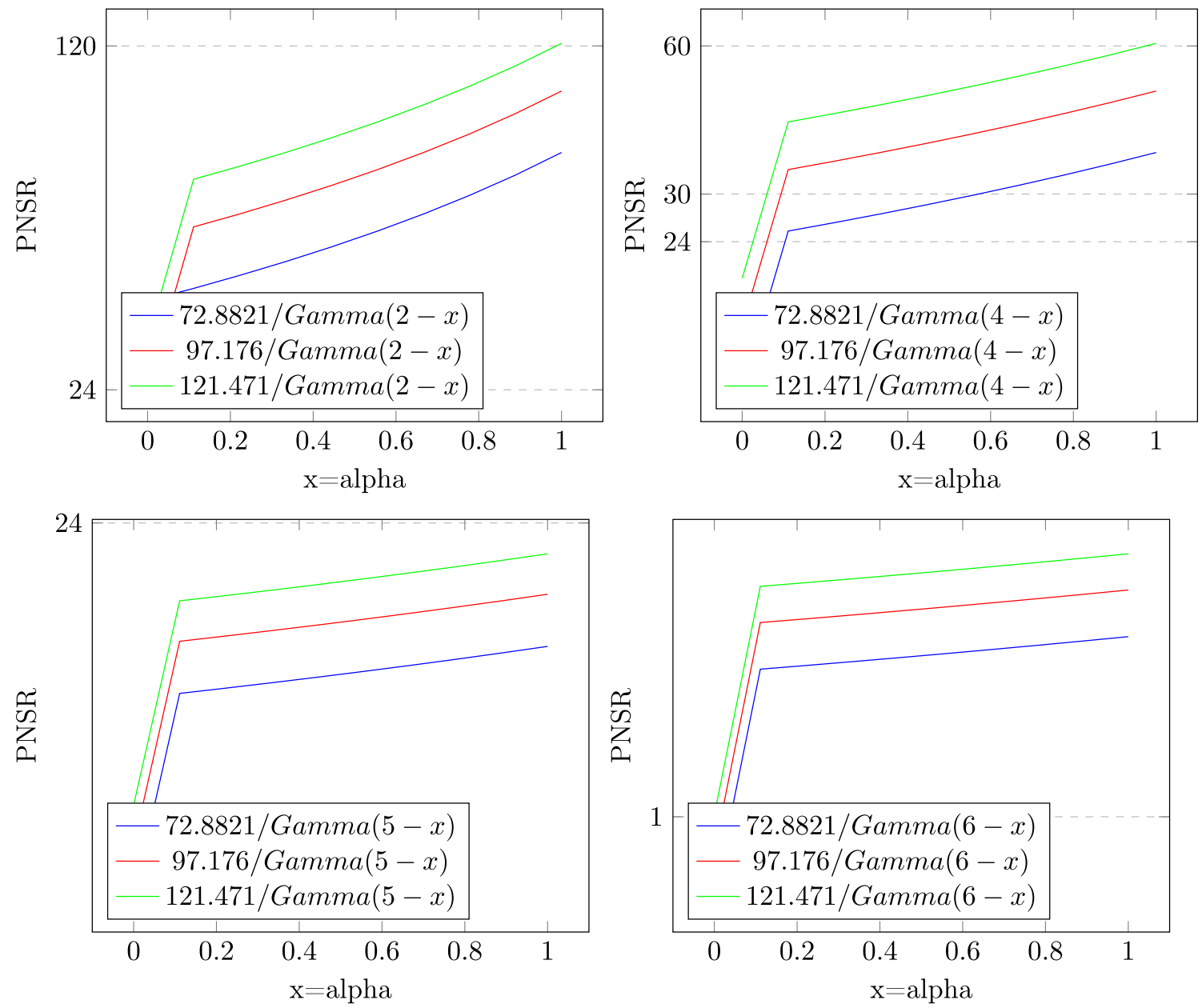

Fig. 4 The relation between $\alpha$ and PSNR for three iterations:15 (blue), 20 (red), 25 (yellow). The first row contains $\sigma^{\alpha}(1,1)$ and $\sigma^{\alpha}(2,2)$ respectively. The second row contains $\sigma^{\alpha}(3,2)$ and $\sigma^{\alpha}(3,3)$ respectively. The best value of PSNR is for $\alpha \in(0.5,0.73)$

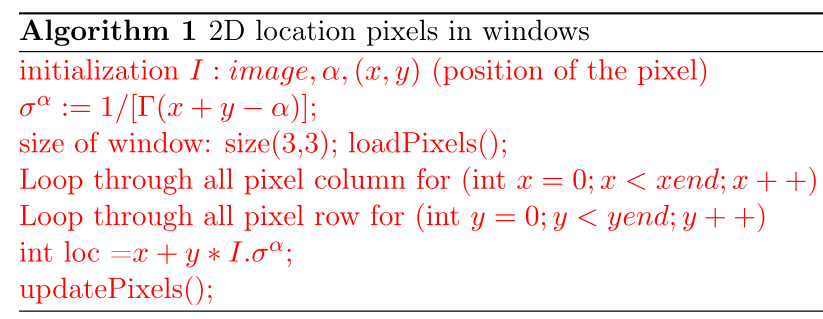

\section{Experimental outcomes}

In this section, we apply the denoising presentation of CFC operator. Presentation assessments were employed by means of Mathematica 11.2. The two groups of images engaged two grayscale images, and two color images. The window's mask of proposed CFC is deliberated to be functioned with a $3 \times 3$ pixels' window. The assessment performances of CFC were designed by both PSNR, and RMSD measures. The PSNR values for the altered values of $\alpha$, are described in Fig. 4, when operating the recommended CFC process image with Gaussian noise $\sigma$ values of 15,20 , and 25 . A big value of $\alpha \in(0.5,0.7)$ matches to a dramatic reduction in PSNR, and vice versa. Consequently, the ideal value of $\alpha$ represents the trade-off between $\alpha$ and PSNR which is required for removing the image noise. In this study, the value of $\alpha$ has been chosen in the interval $(0.5,0.75)$ as shown in Fig. 4 . Since the RMSD is an increasing function with respect to $\alpha$ (see Fig. 5), therefore we conclude our results depending on PSNR.

The outcomes of three images are illustrated in Fig. 6 for iteration equals to 10 (for $\alpha=0.5,0.6,0.7$ ) and Table 1 for the iterations $15,20,25$ and 30 with the fractional value $\alpha=0.6$ (because the best PSNR at $\alpha=0.6$ for all the experience images). The first row displays the original images and the three rows refer to the CFC method for different fractional order. It exhibits that the proposed CFC algorithm offers better performance for all images with deep texture at $\alpha=0.6$, which congruent with the statistical analysis in Fig. 4. The proposed CFC algorithm eliminates 
the noise professionally and keeps the image texture specifics (data, information). This because the fractional calculus allows outmoded initial and edge settings to be involved in the preparation of the processing. Moreover, the fractional calculus implies a historical information with deep knowledge for the image. Fractional calculus

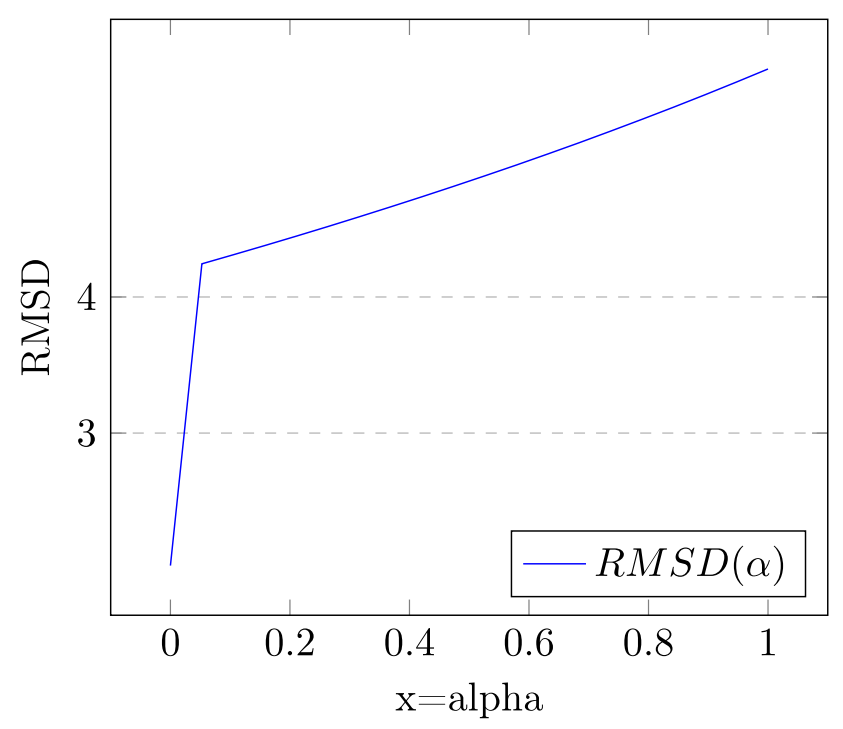

Fig. 5 The RMSD with respect to $0<\alpha<1$ is an increasing function created approaches delivering further degree of freedom in optimization performance, which is very important test in image processing. Not astonishingly, fractional calculus established procedures have utilized in image processing area. Image without texture ignored these facilities (see the cloud image and blood cells image in Fig. 9).

The PSNR and the RMSD values of the four tested images corrupted by two levels of Gaussian noise ( $\sigma=15$, $20,25)$ are shown in Fig. 6 . In addition, Fig. 6 illustrates the numerical assessments of the proposed CFC algorithm. The qualitative results illustrate that the proposed CFC algorithm achieves the superior PSNR and RMSD values compared with the Gaussian smoothing filter.

CFC can be viewed as an iterated guided filter (IGF), which is an explicit filter of the image. This filter contains the guidance image $\Upsilon$, an input image $\eta$, and an output image $\rho$. Both $\Upsilon$ and $\eta$ are introduced beforehand based on the application, and they can be the same image. The filtering output

Table 1 (RMSE,PSNR) of CFC (for $\alpha=0.6$ ) with different iterations

\begin{tabular}{lllll}
\hline Images & 15 & 20 & 25 & 30 \\
\hline Flowers & $(4.30,34.89)$ & $(4.4,34.77)$ & $(4.44,34.4)$ & $(4.6,34.04)$ \\
City & $(2.7,39.27)$ & $(2.2,40.76)$ & $(2.9,38.94)$ & $(3.0,39.20)$ \\
Saturn & $(1.41,45.2)$ & $(1.44,44.86)$ & $(1.9,44.30)$ & $(1.98,43.89)$ \\
\hline
\end{tabular}

Fig. 6 Results by CFC and Gaussian filters noise 10

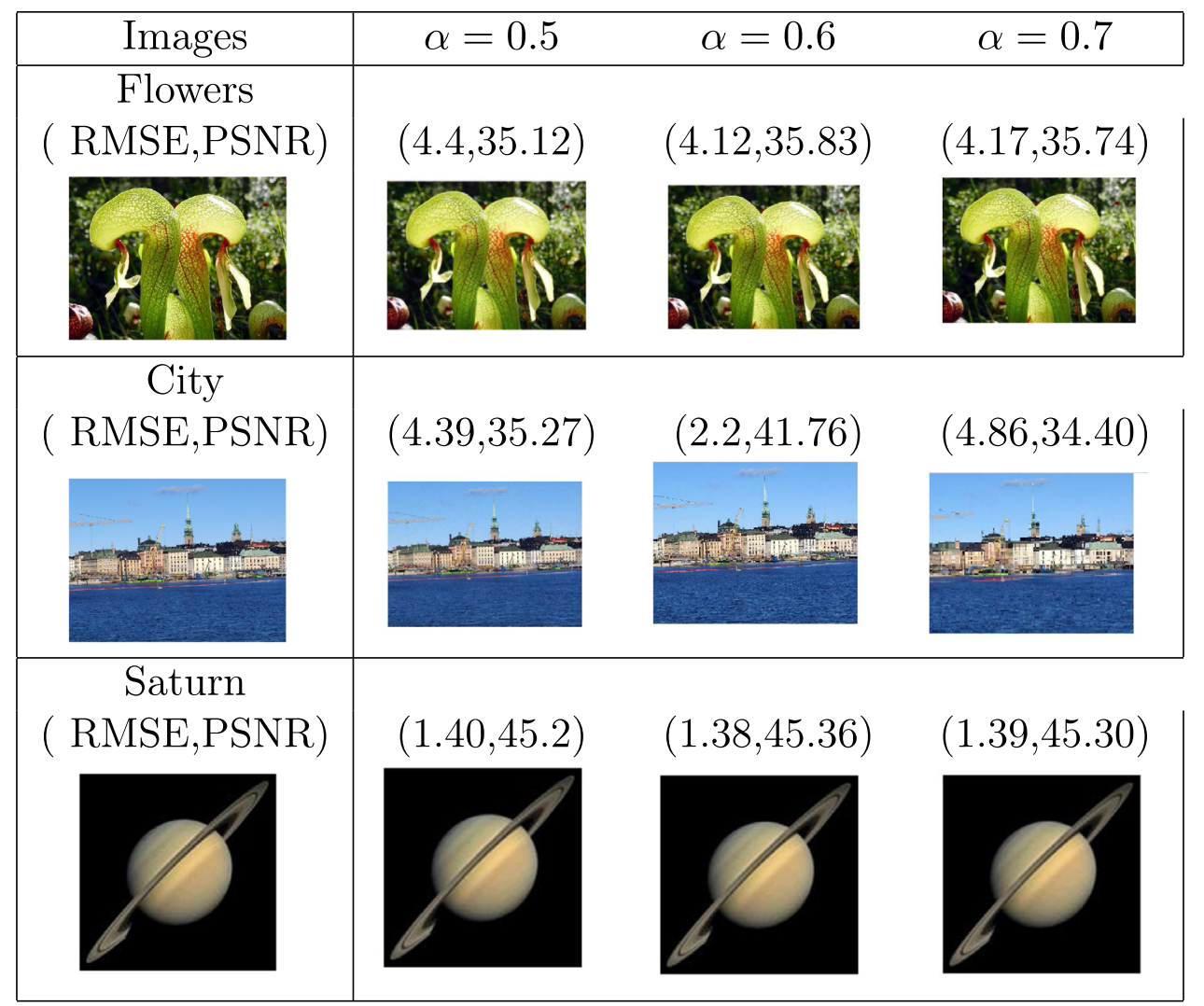


Fig. 7 In this example the guidance $\Upsilon$ is identical to the input $\eta$ with nlter $=60$. The fractional power $\alpha=0.6$

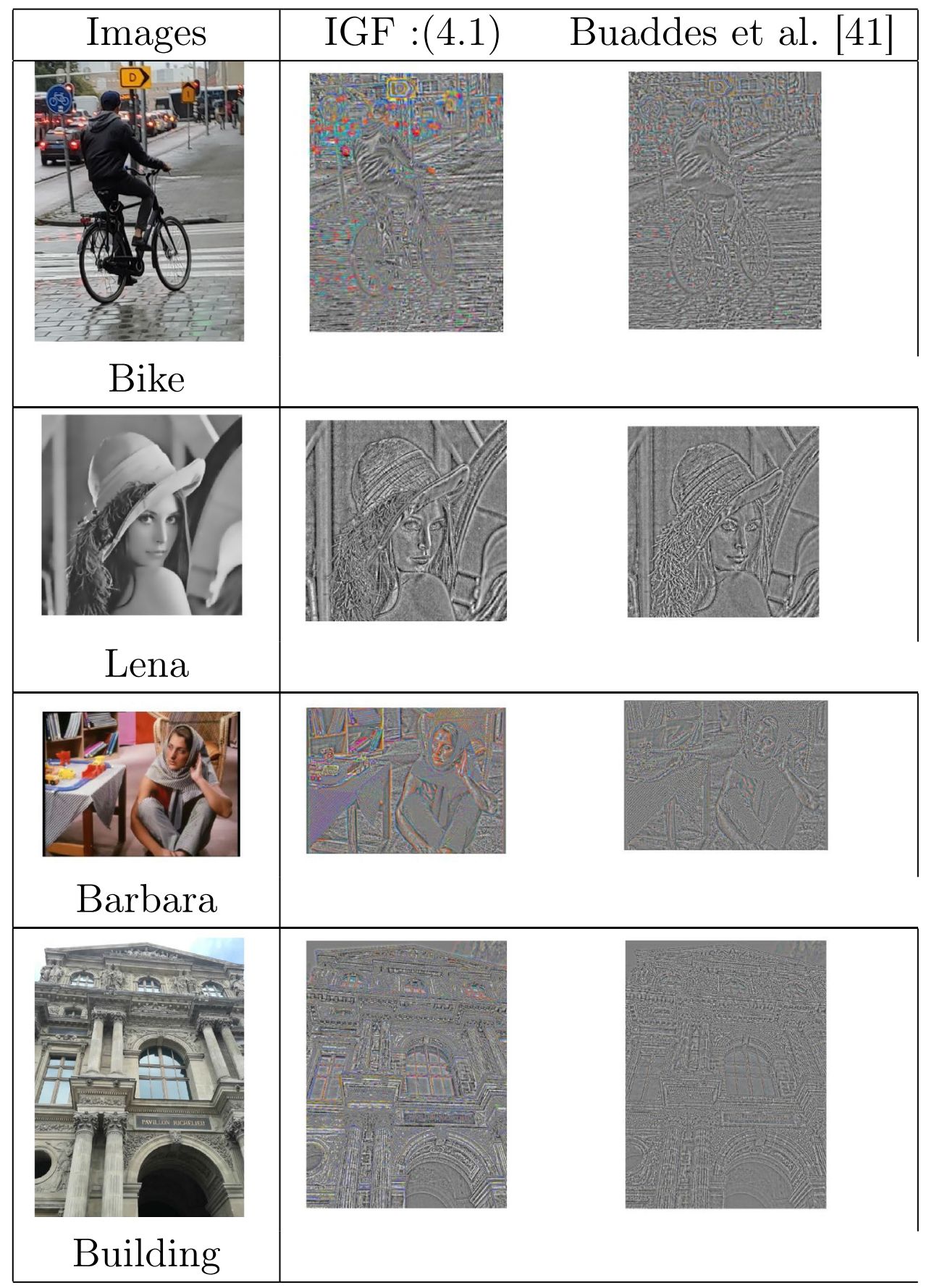

at a pixel $\rho$ is formulated as a weighted average for the pixel $l$ :

$\rho_{l}^{\alpha}=\sum_{J} \sigma^{\alpha}(l, J) \eta$

where $\sigma^{\alpha}(l, J)$ considered as a weighted preparation ( see (3.7)) such that $\sum_{J} \sigma^{\alpha}(l, J)=1$ (see Table 1). For our result, we used the formula

$$
\begin{aligned}
1 / \Gamma(3-0.6)= & \sum_{k=0}^{\infty}(0.8)^{k} \\
& \times \sum_{j=0}^{k}\left((-1)^{j} \pi^{-j+k} \sin (1 / 2 \pi(-j+k+2))\right. \\
& \times \Gamma^{j}(1 /(j !(-j+k) !)) / \pi \approx 0.8 .
\end{aligned}
$$




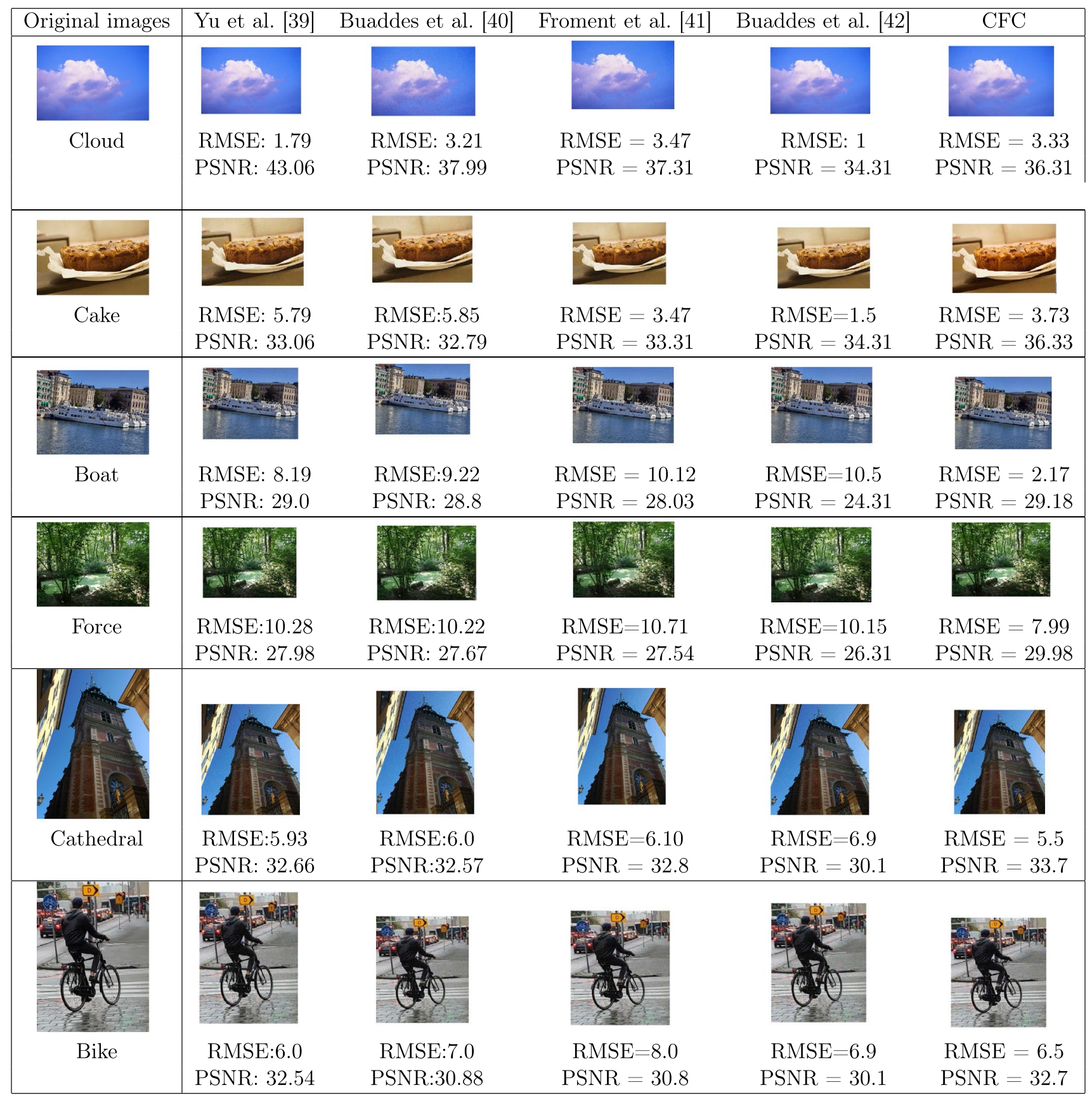

Fig. 8 Results by CFC and different filters

\section{Comparisons}

To check the strength of the proposed CFC algorithm, we compare the proposed study with other approaches for image denoising given in [39-42]. Figure 8 illustrates the results of the proposed CFC algorithm with different denoising algorithms for the "Cloud, Cake, Boat, Force, Cathedral", and "Bike" images with noise $\sigma=15$. In [39], The procedure deals with the image pixel association in the special dimension as well as in the color dimension. The color frequencies of an image are first decor connected with a 3-point orthogonal transform. Each channel is denoised distinctly via local discrete cosine transform thresholding. In [40, 41], the authors generalized non-local means for removing noise in images. While, in [42], the authors regularized and weighted the pixel images by using some optical flow methods (Fig. 7). 


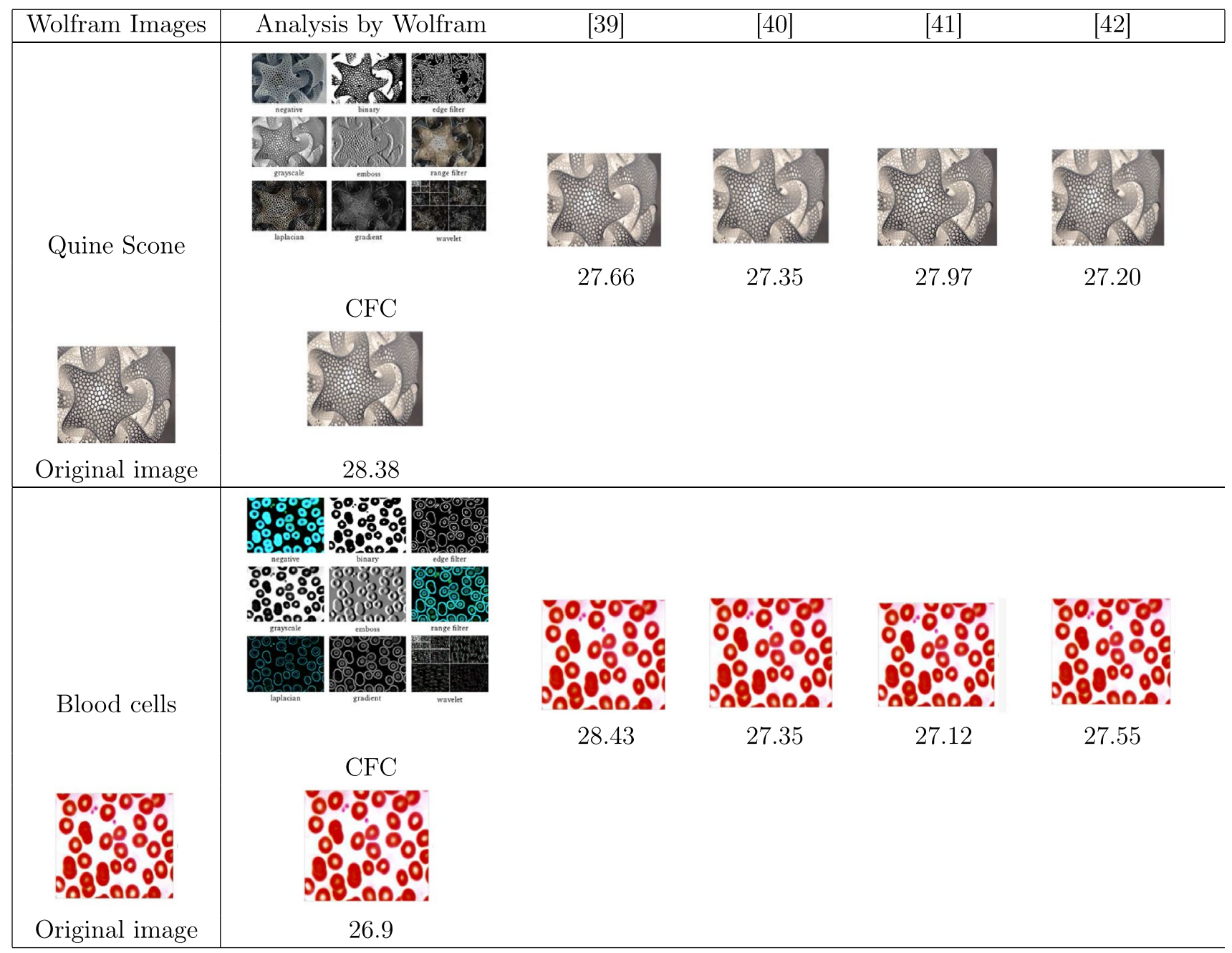

Fig. 9 PNSR using Wolfram images

Figure 8 provides a general performance of different methods in image processing. Figure 8 shows that for image "Boat", the PSNR values for the proposed CFC algorithm are slightly larger compared with the three standard methods for the noise standard deviation $\sigma$ of 15, 25. The proposed CFC algorithm offered satisfactory results (Fig. 9).

\section{Conclusion}

A new image denoising filter based on conformable fractional calculus for image denoising is illustrated to eliminate Gaussian noise. The visual perception results with the PSNR and SSIM results are used to evaluate the denoising performance of a proposed CF-process. Four standard test images were used to validate the performance of the proposed CFC algorithm compared with available filters. The proposed CFC algorithm has shown a good PSNR and RMSE results for all four testing images corrupted by Gaussian noise. In future works, we plan to apply CFCprocess for different image processing applications like texture enhancement of digital images.

Moreover, the CFC - process is a novel serves the development. Corresponding to the other fractional mathematical techniques (fractional differential equations, fractional operators: derivative and integral, fractional special functions, etc.), the CFC fulfills the properties of a fractional derivative. Thus, the CFC - process is the physical creation of image processing technique, because of its controller term. It can deal with indications that feature non-linear, no fundamental, no lowest phase system, non-Gaussian and combine the additive noise with weight linearly. Texture-enhancing is a small part of applications for CFC-processing.

Specific tests showed on the artificial texture images designate the efficiency and proficiency of the suggested method. Visual impresses establish that the offered process achieves well in stabilizing texture specifics and edge 
data of test images. Also, the numerical processes, such as PSNR and SSIM, indicate that the offered technique overall owns more an extraordinary ability of noise decrease than some other traditional approaches.

Acknowledgements The author would like to thank both anonymous reviewers and editor for their helpful advice.

\section{Compliance with ethical standards}

Conflict of interest The author declares that there is no conflict of interest.

\section{References}

1. Bioucas-Dias JM, Figueiredo MAT (2010) Multiplicative noise removal using variable splitting and constrained optimization. IEEE Trans Image Process 19:1720-1730

2. Yao W et al (2019) Multiplicative noise removal for texture images based on adaptive anisotropic fractional diffusion equations. SIAM J Imaging Sci 12(2):839-873

3. Shi J, Osher S (2008) A nonlinear inverse scale space method for a convex multiplicative noise model. SIAM J Imaging Sci 1:294-321

4. Zhou Z, Guo Z, Dong G, Sun J, Zhang D, Wu B (2015) A doubly degenerate diffusion model based on the gray level indicator for multiplicative noise removal. IEEE Trans Image Process 24:249-260

5. Zhang J, Chen K (2015) A total fractional-order variation model for image restoration with nonhomogeneous boundary conditions and its numerical solution. SIAM J Imaging Sci $8: 2487-2518$

6. Jalab HA, Ibrahim RW (2015) Fractional conway polynomials for image denoising with regularized fractional power parameters. J Math Imaging Vision 51:442-450

7. Jalab HA, Ibrahim RW (2015) Fractional Alexander polynomials for image denoising. Signal Process 107:340-354

8. Jalab HA, Ibrahim RW (2013) Texture enhancement based on the Savitzky-Golay fractional differential operator. Math Probl Eng 149289

9. Jalab HA, Ibrahim RW, Ahmed A (2017) Image denoising algorithm based on the convolution of fractional Tsallis entropy with the Riesz fractional derivative. Neural Comput Appl 28:217-223

10. Ibrahim RW, Hasan AM, Jalab HA (2018) A new deformable model based on fractional Wright energy function for tumor segmentation of volumetric brain MRI scans. Comput Methods Progr Biomed 163(2018):21-28

11. Al-Shamasneh $A$, Jalab HA, Palaiahnakote $S$, Obaidellah UH, Ibrahim RW, El-Melegy MT (2018) A new local fractional entropybased model for kidney MRI image enhancement. Entropy 20(5):344. https://doi.org/10.3390/e20050344

12. Yousef H, Ali R (2002) Spatially adaptive multiplicative noise image denoising technique. IEEE Trans Image Process 11(12):1397-1404

13. Rudin L, Pierre-Luis L, Stanley O (2003) Multiplicative denoising and deblurring: theory and algorithms. Geometric level set methods in imaging, vision, and graphics. Springer, New York, pp 103-119

14. Bioucas-Dias JM, Figueiredo MAT (2010) Multiplicative noise removal using variable splitting and constrained optimization. IEEE Trans Image Process 19(7):1720-1730
15. Steid G, Teuber T (2010) Removing multiplicative noise by Douglas-Rachford splitting methods. J Math Imaging Vision 36(2):168-184

16. Li B, Xie W (2016) Image denoising and enhancement based on adaptive fractional calculus of small probability strategy. Neurocomputing 175:704-714

17. Yao W, Guo Z, Sun J, Boying W, Gao H (2019) Multiplicative noise removal for texture images based on adaptive anisotropic fractional diffusion equations. SIAM J Imaging Sci 12(2):839-873

18. Guo Z, Yao W, Sun J, Boying W (2019) Nonlinear fractional diffusion model for deblurring images with textures. Inverse Probl Imaging 13(6):1161

19. Mei J-J, Dong Y, Huang T-Z (2019) Simultaneous image fusion and denoising by using fractional-order gradient information. J Comput Appl Math 351:212-227

20. Mei K, Dongdong H, Binjie Q (2019) Adaptive fractional-order differentiation filter guided by feature asymmetry for featurepreserving ultrasound despeckling. In: Eleventh international conference on digital image processing (ICDIP 2019), vol 11179, p 111793H. International Society for Optics and Photonics

21. Zhang Y-D, Wang S-H, Liu G, Yang J (2016) Computer-aided diagnosis of abnormal breasts in mammogram images by weighted-type fractional Fourier transform. Adv Mech Eng 8(2):1687814016634243

22. Nandal A, Dhaka A, Gamboa-Rosales H, Marina N, Galvan-Tejada Jl, Galvan-Tejada CE, Moreno-Baez A, Celaya-Padilla JM, LunaGarcia H (2018) Sensitivity and variability analysis for image denoising using maximum likelihood estimation of exponential distribution. Circuits Syst Signal Process 37(9):3903-3926

23. Yang $X$, Peng $X$, Jin H, Zhang J (2019) Low-rank tensor completion with fractional-Jacobian-extended tensor regularization for multi-component visual data inpainting. Digit Signal Process 95:102571

24. Aubert G, Aujol J-F (2008) A variational approach to removing multiplicative noise. SIAM J Appl Math 68:925-946

25. Hu J, Pu Y, Zhou J (2011) A novel image denoising algorithm based on Riemann-Liouville definition. J Comput 6:1332-1338

26. Jalab Hamid A, Ibrahim Rabha W (2013) Texture enhancement for medical images based on fractional differential masks. In: Discrete dynamics in nature and society

27. Ibrahim R, Jalab HA (2015) Image denoising based on approximate solution of fractional Cauchy-Euler equation by using complex-step method. Iran J Sci Technol (Sciences) 39:243-251

28. Salahat E, Murad Q (2017) Recent advances in features extraction and description algorithms: a comprehensive survey. In: 2017 IEEE international conference on industrial technology (ICIT), pp 1059-1063. IEEE

29. Hassaballah M, Ismail AA (2016) Detection and description of image features: an introduction. Image feature detectors and descriptors. Springer, Cham, pp 1-8

30. Hassaballah $M$, Amin AA, Hammam AA (2016) Image feature detectors and descriptors: foundations and applications. Springer, New York, pp 11-45

31. Pan J, Ren W, Zhe H, Yang M-H (2018) Learning to deblur images with exemplars. IEEE Trans Pattern Anal Mach Intell 41(6):1412-1425

32. Ancuti CO, Ancuti C (2013) Single image dehazing by multi-scale fusion. IEEE Trans Image Process 22(8):3271-3282

33. Khan SA, Ishtiaq M, Nazir M, Shaheen M (2018) Face recognition under varying expressions and illumination using particle swarm optimization. J Comput Sci 28:94-100

34. Nazir M, Jan Z, Sajjad M (2018) Facial expression recognition using histogram of oriented gradients based transformed features. Clust Comput 21(1):539-548

35. Munir A, Hussain A, Khan SA, Nadeem M, Arshid S (2018) Illumination invariant facial expression recognition using 
selected merged binary patterns for real world images. Optik 158:1016-1025

36. Anderson DR, Ulness DJ (2015) Newly defined conformable derivatives. Adv Dyn Syst Appl 10(2):109-137

37. Abdeljawad T, Alzabut J, Jarad F (2017) A generalized Lyapunovtype inequality in the frame of conformable derivatives. Adv Differ Equ 2017(1):321

38. Zhou HW, Yang S, Zhang SQ (2018) Conformable derivative approach to anomalous diffusion. Phys A Stat Mech Appl 491:1001-1013

39. Yu G, Sapiro G (2011) DCT image denoising: a simple and effective image denoising algorithm. Image Process Line 1:292-296
40. Buades A, Coll B, Morel J-M (2011) Non-local means denoising. Image Process Line 1:208-212

41. Froment $J$ (2014) Parameter-free fast pixelwise non-local means denoising. Image Process Line 4:300-326

42. Buades A, Lisani JL (2018) Video denoising with optical flow estimation. IPOL J 8:142-166

Publisher's Note Springer Nature remains neutral with regard to jurisdictional claims in published maps and institutional affiliations. 